\title{
Field Testing a Rare Species Bioacoustic Smartphone Application: Challenges and Future Considerations
}

\author{
Nadia Pantidi, Stuart Moran, \\ Khaled Bachour, Tom Rodden \\ Mixed Reality Lab \\ University of Nottingham, \\ Nottingham, UK \\ \{firstname.lastname\}@nottingham.ac.uk
}

\author{
Davide Zilli, Geoff Merrett \\ and Alex Rogers \\ Electronics and Computer Science \\ University of Southampton, \\ Southampton, UK \\ \{dz2v07;gvm;acr\}@ecs.soton.ac.uk
}

\begin{abstract}
New Forest cicada is a declining species native to the UK, and the last unconfirmed sighting was in 2000. One of the difficulties in identifying the cicada is that it sings at a high frequency typically inaudible to adults. In this paper we describe a field test of a novel citizen science smartphone application designed to detect and classify the cicada's call. We discuss some of the obstacles to studying this novel technology, and describe the results from a user trial with a simulated cicada. Our observations are then used to inform a series of design considerations for those developing a similar class of application, and improvements for the application itself.
\end{abstract}

Keywords-app; acoustics; biodiversity; cicada; field test; new forest; simulation; smartphone;

\section{INTRODUCTION}

In the field of biodiversity surveying is a major part of tracking, studying and conserving species and landscapes. One of the problems professionals face are significant gaps in their data, predominately due to resource constraints. Even with volunteers, it can often be difficult to get quality data due to the skills required for rigorous identification. In this paper, we focus on the surveying of a type of cicada native to the New Forest National Park in Southampton, UK. The cicada has not been seen for over 10 years, and is difficult for humans to detect due to its small size and singing at an inaudible frequency. This motivated us to develop a smart phone application to assist in surveying [1]. The application utilizes the phone's onboard microphone which can record high frequencies, and the processing power to run classifiers which automatically detect the species.

An interesting challenge during the development of the application was to evaluate user interaction with the technology. The major problem for trialing the app was the availability and accessibility of the New Forest cicada (NFC). To combat this unique constraint, an electronic cicada was developed which emits a clean and pure frequency similar to that of the NFC. This electronic NFC (eNFC) allowed us to study user interaction with a prototype of the application, without the need for a cicada; thereby allowing us to safely study the app without endangering the NFC or the forest. We present results from a field trial using a prototype of the application and eNFC. We observed a number of key interactions with the prototype application that may have endangered the forest, while also de-motivating participants. From this, we suggest a series of improvements for the application itself as well as design considerations for those intending to develop a similar class of application.

\section{BACKGROUND}

There are many different applications and possibilities for/when collecting data through a microphone; particularly detecting distinctive and predictable species' sounds in the natural world [2]. There are a number of projects which make use of the proliferation of smartphones and their onboard microphone to classify sounds which identify the place [3] or assess levels of noise pollution [4]. However, much of this work relies on standalone software for post processing such as RAVEN [5]. With this constraint in mind, there is a trend in utilizing the available computational power on smartphones for more localized classification. This type of automated classification is a paradigm shift for many naturalists [6], which highlights the need to better understand the implications and impact of the technology. As a first step towards this, we consider the wider social impact of citizen science technology on both curators and participants.

\section{A. Social Implications of Citizen Science}

There are three main factors widely acknowledged by practitioners as the key to participant acceptance in citizen science: data quality, privacy and motivation [7]. Data quality and scope is heavily determined by the skill of the participants [8], their levels of enthusiasm and the coverage of designated area [2]. Experts in the community have been known to place doubt on the quality of data collected by amateurs, and hence its validity for academic research [7],[8],[9].

Privacy is an important consideration when using mobile systems for citizen science, particularly given the potential for automated audio, location and image collection [2]. One difficulty when protecting privacy (e.g. anonymity) is the inability to assess the trustworthiness and reputation of the citizen; which as a consequence, creates problems for data quality [10]. Motivation is a challenge in citizen science, with repetitive tasks often leading to boredom [11], which stretches the endurance of citizens' patience [9]. Gamification is one successful approach which has been previously adopted in mobile acoustic based projects, and allows users to contribute in a playful, lightweight way [11]. 
Depending on the type of citizen science project, the issues of data quality, privacy and motivation manifest in different ways. For example, participatory sensing can create tension in a project between its educational and scientific goals [12], between authenticity of the data and privacy invasion [10], and between data quality and learning [13]. In contrast, opportunistic sensing (automatic detection) can help alleviate these issues, but comes at the cost of losing users' full engagement with the data collection activity [2], and hence their education based motivation.

There are also a number of additional issues that can affect citizen science that should be considered. In biodiversity, there are networks of trust through the establishment of communities of practice, where both data credibility and potential for data misuse can be monitored and controlled [14],[15]. However, this becomes an issue with citizen science projects in this domain as trust weakens when members of the general public, who are external to these communities of practice, begin contributing to the projects. For example, some projects have been viewed as politically motivated, and subsequently treated with suspicion and distrust by participants [16]. Equally, participants who collect data have been known to maliciously pollute it for their own benefits [17]. Limited control over data collection could also lead to 'over-collection' of a species, destruction of sensitive areas in the environment or biased use within environmental politics for decision making [15]. This has led to masking locations of rare species or protecting land owners property [14].

The sharing of citizen science data is a debated point where some practitioners view the benefits of sharing information as outweighing any possible negatives [15], [16]. For example, one suggested benefit of the use of this information is for citizens to manipulate habitats and positively intervene in ecosystems which could have powerful ecological and social impact [18]. However, when intensive post-processing of the data is needed, researchers are less likely to share the data [19]. This raises ethical questions surrounding the ownership of the data, how it should be handled and who are the decision makers regarding this process [20]. Finally, problems can arise from participants engaging in activities which target popular species, limiting the inferences that can be made about the wider ecosystems [21]. For example, birds and amphibians are considered indicator species for assessing environmental health [6], which creates additional value for their surveying. This brings to question how potential participants' time should be most effectively used when surveying. Such issues with established citizen science projects are expected to be equally or more evident when novel technologies (such as mobile sensing) will be used for the same purposes and it is important that these are considered.

\section{THE NEW FOREST CICADA}

A cicada is a type of flying insect in the order Hemiptera, and can be found all over the world. The New Forest cicada (Cicadetta montana s. str.) is the only cicada native to the UK. Unfortunately, the species is suffering from a general decline, as evidenced by the last unconfirmed sighting in 2000. There are many challenges for professionals surveying this cicada, which have resulted in gaps in our knowledge of the prevalence of the species.

- Time frame: The cicada lives underground for up to eight years as a larva, before taking on its adult form for a short four to six weeks period between May and July.

- Population size: This species of cicada is not periodical and so adulthood is not synchronised, thus decreasing the potential population numbers at any one time.

- Habitat size: The New Forest is approximately $600 \mathrm{~km}^{2}$, making it difficult to survey effectively.

- Weather conditions: The cicada only emerges in certain weather conditions (sunny, calm and above $20^{\circ} \mathrm{C}$ ).

- Means of identification: The cicada is difficult to identify at a distance, as it sings at a frequency of 12$14 \mathrm{kHz}$, which is at the limits of hearing for most adults.

These distinct challenges in ecological surveying motivated the surveyors to explore what technological solutions might be available to them. Through a series of meetings between the core stakeholders (expert entomologists, and members of the New Forest National Park Authority) and researchers, participatory sensing was agreed as an effective solution. This approach is particularly attractive given the untapped potential of $\sim 13$ million day-visits per year.

\section{A. Mobile Phone Application}

Ever increasing smartphone ownership amongst the public led to the appeal of a phone-based participatory sensing tool for the cicada. Through a number of trials, it was discovered that a typical onboard microphone was able to pick up the frequency of the cicada. An algorithm was then developed for acoustic classification (for more details see [1]), which can accurately distinguish and detect the singing of the NFC. Furthermore, given the computational power available on a smartphone it can be run locally. The algorithm was trained using audio recordings from Slovenia where the same species of cicada is still abundant. With the core technology built in the form of a prototype application, it was necessary to explore how users would interact with the application.

\section{B. Challenges for Field Testing}

One common methodological approach in the field of human computer interaction is rapid prototyping [22], which often involves deploying and testing the technology in situ. This allows designers to explore issues relating to the users' interactional experience earlier on in the development. One of the main reasons for adopting this approach is the unique problem of the time frame for deployment and evaluation. With the cicada only 'in season' during the summer months there is limited opportunity to test the application and introduce changes. With rapid testing, any necessary changes can be introduced before the cicada season begins.

Although this approach is well suited to speedy development, there are unfortunately a number of intriguing issues which make this type of study problematic. For example, while testing in situ is necessary to generate more realistic user behaviors, this is also a high risk strategy as the forest environment itself is a sensitive entity. To avoid damage to 
species on the ground and the environment from trampling, testing should aim to be in a more controlled environment.

However, if studies are not conducted in the forest, how can the application be tested for exploring the New Forest cicada? Even more important is the consideration that the cicada is a very rare species, so how does one study the detection of a rare species in situ if we cannot find an example of the species to test with, let alone in the correct season. In short, during field testing of the app it is necessary to protect the forest and its inhabitants, and also devise a mechanism to emulate the NFC without the need for an actual specimen.

\section{Electronic New Forest cicada}

To support the process of rapid-prototyping a set of electronic new forest cicadas (eNFC) were developed. These eNFCs were constructed on small printed circuit boards, and designed to emit a constant acoustic square wave between $15 \mathrm{kHz}-18 \mathrm{kHz}$ (four cicadas, each with distinctive frequencies). This approach allows us to strategically place versions of the cicada in a forest like environment in a controlled way. Hence, this allowed us to minimize the potential damage to the environment during testing, while also allowing us to explore the interaction of users when they do (and do not) find the cicada. Testing participatory sensing in this way contributes toward the sustainability of the environment and the cicada.

\section{Cicada Prototype Game}

Using the eNFCs, we developed a game to explore how users interacted with the prototype application. The need for a game was to make the experience as engaging as possible. The game asked players to search the environment for four different electronic cicadas, each representing a different frequency and colour. When an electronic cicada is detected, the app vibrates and the colour of the associated cicada lights up permanently on the screen.

Each electronic cicada was housed inside a paper origami cicada to help visually identify it (for children). As a part of this game, a prototype sonogram visualisation was used. A sonogram is a visual representation of fluctuations in frequency and amplitude of sound, and is frequently used by professionals for analysing species sound. For this reason it was included in the app, but was animated in such a way to
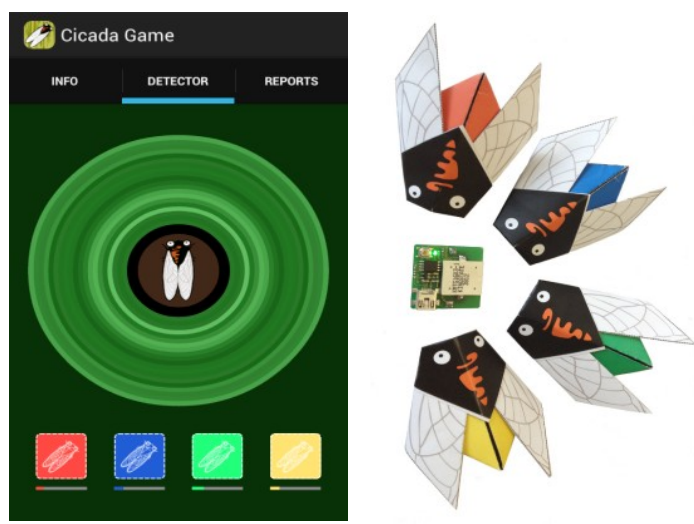

Fig. 1. Prototype cicada Mobile App and Electronic cicadas emulate popular mobile applications used by members of the public which perform acoustic analysis (e.g. Shazam www.shazam.com/).

\section{STUDY DESIGN}

With the prototype application built, and an engaging interaction designed, it was possible to develop a study to simulate and safely explore user interaction and experience. The campus of our university was chosen as the study location, first because it was practical, and because it has a representative mix of environments also found in the New Forest, albeit on a much smaller scale, including dense areas of foliage, a lake, public areas and paths and more sensitive areas with wildlife habitats. To examine all facets of usability, we needed participants to find at least one eNFC (which did not always occur) and so four electronic cicadas were placed in a variety of areas around the campus. The eNFCs were carefully placed across a large area and in locations that matched actual Cicada habitats (i.e. deep in the foliage, high in trees). Thus making them difficult, but not impossible, to find, as would be the case in the real forest conditions.

Two user trials were run: one with six participants working in pairs sharing one smart phone; the other with six participants searching for cicadas individually. This was designed such that we could explore the different interaction arrangements that might take place when the app is deployed e.g. families/couples and individuals. Snowball sampling was used to recruit participants, which led to an age range of $23-40$, with a mixture of students and professionals; which represents the public demographic targeted by the app.

In each trial, participants were asked to use the cicada game mobile app to find as many cicadas as they could on campus within 30 minutes. They were given a map of the area, which included a boundary indicating the general area the cicadas were placed. Participants were shadowed and video-recorded by the researchers. Once the game time elapsed, participants were instructed to return to the start location where they were interviewed individually regarding their overall experience and the specifics of the mobile app interface. Also, a demonstration of a more advanced version of the app which was under development was shown to them for feedback.

\section{RESULTS}

The following results comprise predominately of interviews with participants reflecting on their experience, along with some observations of the app in use.

\section{A. Uncertainty and Interpretation}

During the trial, participants showed uncertainty about a number of different characteristics of their interaction with the app. For example, there was confusion over the detection accuracy and range:

"I wasn't aware how close you actually have to be to these things "

"What is the range? How close do I need to be to pick it up?"

When unsure about a detection occurring, users would tap the various tabs and icons on the application and buttons on the phone itself in a way that is typically indicative of a device 
perceived to be broken. There was also puzzlement about the meaning of the sonogram:

"I actually thought this was a kind of the hot and cold thing, so as you got closer to it, it would pulse more frequently and more intensely"

"Ummm so it seems that when something is louder, there's more color and l'm wondering if sort of the diameter of this might be related to either the frequency, or the distance... but l'm not quite sure"

As a consequence, this influenced its perceived value in searching for and finding the cicada. While the majority of participants had misguided expectations and assumptions, some understood the sonogram, but still questioned its value.

"Actually I think that it makes sense, because it gives the noise frequency, and these circles are just the frequency, so this one is on all the time... but I don't think it's got any use [...] It reacts to any noise, so it doesn't make any useful feedback to us

As part of the study design we consciously chose not to explain the workings of the application so that we could observe participants' salient expectations and assumptions. This revealed some of the non-intuitive aspects of the interface, such as what to expect from detection:

"It would be useful to make some instruction what happens when you detect the cicada"

This type of information would help verify that the application is actually working, and build confidence in its accuracy (thereby alleviating the previously established confusion).

"I think some tutorial would be nice, when people use it first, to like, to prove it really works... so they have proof"

One participant suggested that real-time instructions on approaches to detecting the cicada would be of use:

"Maybe if it gave me some hints, like I saying, for example if it said ok, she's spent 10 minutes and only found one, she clearly needs some help"

\section{B. Motivation and Engagement}

With any citizen science endeavor, the participants' motivations are of critical important to its success. In the case of the cicada app, it became clear that participants' motivations were limited by frequent negative results received during the trial.

"If there were things that you find in your own back garden, so you know you're not going to be disappointed, there's always going to be, there's always the chance that you'll find something, ummm cause to get a report that says nothing found, it just leaves, it could leave people feeling a little cold and empty"

"I don't have too much incentive if I can't find anything during a period like several days"

In relation to this, participants felt that some sort of interactivity was lacking in the application, in addition to the limited number of species being detected, thus lowering potential engagement:

"I think to keep up the engagement it needs to be ummm more interactive, more than anything"

"It needs a bigger range, otherwise it's boring"

Participants also spoke of increasing motivation through an understanding of what others were doing, perhaps in the form of a map and more explicit details on their contribution to science. More localized feedback was also suggested to track personal progress and motivate:

"How the big picture looks like, and some spots that I can see, maybe I can see something, because somebody finds something in that region, maybe I can make some guess, so make like, I'm actually participated in the actually scientific act, not just doing some stupid things blindly, searching for stuff"

"It's nice to have feedback, so you start feeling you're a part of a community"

However, in some cases, participants explained that they simply had no interest in biodiversity and by extension in the cicada application:

"I don't think l'm a sort of insect nerd enough to do this [...] l'm not sure if I would be going out of my way to ya know, dedicated weekends on end to try to find it."

\section{"My mum and dad would, but I wouldn't"}

For many participants visiting the forest for pleasure was described as their primary activity, and some felt that the current of mode of interaction with the app actually interfered with this, hence reducing the desire to use it in the future:

"It's funny how we make apps that work in the forest and when you use them you don't wanna look at the screen [...] the need to focus on what's happening gets in the way of the experience of walking in the forest"

"If it would give you some sort of alarm ummm if there might be a chance that you're close [...] be great if it was vibrating, cause that way I don't have to look at the screen all the time and I can actually look at the environment, which is why I came to that place in the first place"

\section{"There's a conflict with using apps in the forest"}

\section{Teaching and Learning}

The educational benefits of the application were evident in all participant responses:

"I would probably use this more as a sort of teaching tool for a child or so... about how to find insects and some sort of engagement with nature"

"The kids I teach at school would love this"

With respect to teaching and learning, some participants were particularly keen on the addition of specific types of information:

"The time of day, like it might say oh actually you're more likely to find cicadas this time of day because of these reasons"

"Maybe some more pictures as there's quite a bit of writing in some bits of it"

"Maybe if there was more, interaction on this page...so when you find something you could actually find out a little bit about it"

\section{DISCUSSION}

While the study involved a limited number of participants, it revealed a number of issues with respect to the application. We believe these issues, while directly relevant to the cicada application, need to be considered by others developing similar citizen science biodiversity applications. These are discussed below.

\section{A. Complexity of Information}

Among the participants there were different interpretations of the visualizations where some understood their meaning while others misunderstood them. Although, all participants 
agreed that the visualizations were aesthetically pleasing, they questioned their value in finding the cicada. However, despite not finding value in having the sonogram, participants did not see it as detrimental to their experience either. Given the value of the sonogram as both a genuine tool for experts that fully understand it, and also as a means of educating non-expert users about the interpretation of sound, the downside of its inclusion seems minimal in its impact.

\section{B. Confidence and Trust}

While previous work e.g. [7], [15], [17] discusses the trust experts place in the collected data, our study showed issues of trust that might arise from the users' part. In the study we consciously did not show participants how to use the application in order to explore their expectations and assumptions about the use of the application (particularly as we cannot assume real users would read instructions). This caused some difficulty for participants as they were not clear on how to interpret the visuals of the sonogram, and also not sure what to expect when a detection was made.

Without this information, participants began to doubt the accuracy of the application, and question its range of detection, to the point that some began to search visually for cicada. However, there are a number of questions to be answered in this space related to the different types of tutorial and demonstration, and the complexity of the language in the content. For example, the inclusion of a 'demo' button that could show the types of feedback that can be expected to be received, or a simple comic style set of instructions that appear when the application is first loaded might be useful. Still better understanding of the use of the app can increase confidence and trust and needs to be considered.

\section{Motivation and Negative Results}

Not all participants were able to find at least one cicada, and none of the participants managed to find them all. Participants that did not find a cicada were left disappointed, which, as they explained, would likely influence their motivation to use the application. Those who found at least one cicada expressed a sense of instant gratification that eventually subdued as no more cicada were found. The application also lacked a real-time engaging quality, which left some participants bored as they walked around the forest. Participants described a number of possible solutions to help deal with negative results, boredom and also motivate repeated use. There was a clear desire for the detection of insects and animals beyond the cicada, which would give the app a broader appeal. The classification algorithm in our case has the potential for additional insect detections (grasshoppers, crickets) which will help satisfy this need.

Another proposed solution for the motivation issue was the use of maps and personal records, to help track progress and give a sense of a 'bigger picture'. This need to track data could be met through the post-detection web interface, which allows users to see an aggregated set of data - although there may be some minimal information that could be stored and presented locally on the phone (e.g. transects). Similar solutions have been reported by [4], [7], [11] and can promote motivation and engagement, however, in the case of biodiversity apps, designers need to consider how to balance revealing location data against protecting rare species.

Additional content was also seen as a potential tool to stimulate more engagement with the application, such as more detail, and different insects including photographs and sound files. One example given was that when an insect was detected, a picture could pop up providing more information about it. This approach could also be adopted in the form of "Did you know...?" or "Fun Fact 36..." notifications that periodically pop up on the interface. Not only would this create a greater sense of interaction and engagement with the application from the users' perspective, but it would also give them a reason to keep the application open and in hand. Such information could be sourced from experts in the community (a version of this was included in the real application). It could also be locationbased, and trigger locally and contextually relevant information.

\section{Protecting the Forest}

It was very clear from the observations that some participants unreservedly left official paths following desire lines and venturing on to untrodden areas (see Figure 2). In contrast, other participants explicitly stuck to the paths, and asked 'permission' to leave them. During early discussion with stakeholders, they expressed concerns regarding the inclusion of a map in the interface (which was a typical inclusion in participatory sensing projects). Encouraging users to delve deeper in the forest was perceived as an unacceptable risk to the forest. One expert explained that the desire to find the cicada may lead some users to wander into 'unsearched' areas to increase the likelihood of finding.

For this reason a map was not included in the application, but from observations during the trial this still led to people leaving the path. One suggestion might be to include a series of DO's and DON'T's within the applications tutorial. This could encourage users to use the app in a certain way (e.g. to not run with the application), and to not leave the official paths - explaining to them why this is important. This can serve as an educational mechanism, but also help dissolve the experts concerns about path leaving. This feature was included in the real application.

\section{DESIGN CONSIDERATIONS}

In light of this discussion we make some recommendations on the design of smartphone apps for participatory citizen
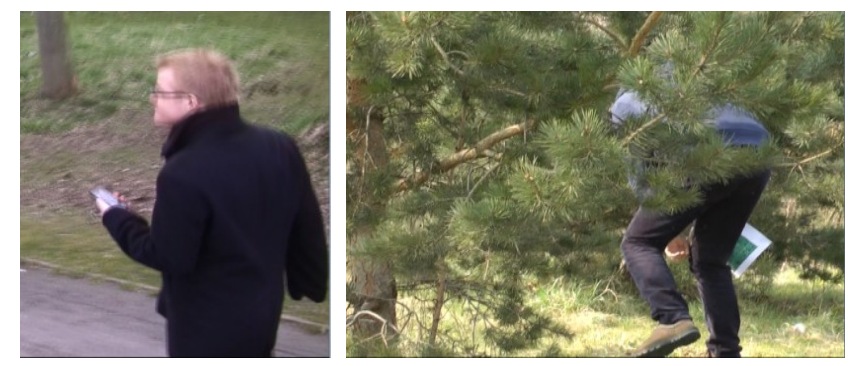

Fig. 2. Participants sticking to and veering off official paths 
science projects. In addition we briefly describe the steps we have taken to follow these considerations in the subsequent version of the app. There is a need for explicit measures to ensure adequate protection of users and the environment they are exploring. While our app avoided encouraging users to leave the designated trail, users still chose to do so. We thus introduced a cartoon that is accessible through the interface of the app which asks that users avoid leaving the trail and explains the dangers of doing so to them.

Including within the interface a feature that is not comprehensible to the average user does not necessarily detract from their experience. While experts can make use of such features, users who do not understand them are able to ignore them as long as they weren't necessary for performing their tasks. As such, the frequency sonogram displayed in this app prototype remained as part of the subsequent version. When the main purpose of the app is unlikely to generate a positive experience, it is necessary to create secondary objectives that give the user a sense of achievement and maintain motivation. These objectives can take different forms such as allowing the user to:

- learn about other types of insects that are detected by the app while using it to look for the cicada,

- track their activity and understand the value of their contribution to the bigger picture.

\section{CONCLUSIONS}

This paper described a study designed to evaluate the usability of a mobile phone application in a citizen science project aimed at rediscovering an endangered species of insect. The nature of the research created certain obstacles: difficulty in testing an app whose target environment is a protected forest and whose target objective is to find an insect that might not be there. We thus simulated the search of the cicada by planting electronic frequency generators in a forest-like environment. Our results highlighted some considerations for the design of such applications; namely, the explicit need to protect the forest, the importance of secondary sources of motivation and the importance of having a prior understanding of how the app works. These design considerations were used to further develop the Cicada Hunt app, which was subsequently launched and studied in the New Forest [23].

\section{REFERENCES}

[1] D. Zilli, O. Parson, G. Merrett, and A. Rogers, "A hidden Markov model-based acoustic cicada detector for crowdsourced smartphone biodiversity monitoring," in Proceedings of the International Joint Conference on Artificial Intelligence, 2013.

[2] A. T. Lane, N. D., Miluzzo, E., Lu, H., Peebles, D., Choudhury, T., \& Campbell, "A survey of mobile phone sensing," Communications Magazine, IEEE, vol. 48, no. 9, pp. 140-150, 2010.

[3] Y. Chon, N. Lane, F. Li, H. Cha, and F. Zhao, "Automatically characterizing places with opportunistic crowdsensing using smartphones," in Proceedings of the International Conference on Ubiquitous Computing, 2012, pp. 481-490.

[4] N. Maisonneuve, M. Stevens, and B. Ochab, "Participatory noise pollution monitoring using mobile phones," Information Polity, vol. 15, no. 1, pp. 51-71, 2010.
[5] C. Cooper and H. Mills, "New software for quantifying incubation behavior from time-series recordings," Journal of Field Ornithology, vol. 76, no. 4, pp. 352-356, 2005.

[6] J. Wimmer, M. Towsey, B. Planitz, I. Williamson, and P. Roe, "Analysing environmental acoustic data through collaboration and automation," Future Generation Computer Systems, vol. 29, no. 2, pp. $560-568,2013$.

[7] S. Kim, J. Mankoff, and E. Paulos, "Sensr: evaluating a flexible framework for authoring mobile data-collection tools for citizen science," in Proceedings of the 17th Conference on Computer Supported Cooperative Work (CSCW), 2013, pp. 1453-1462.

[8] A. Wiggins and G. Newman, "Mechanisms for data quality and validation in citizen science," in e-Science Workshops (eScienceW), 2011 IEEE Seventh International Conference on, 2011, pp. 14-19.

[9] D. Delaney, C. Sperling, C. Adams, and B. Leung, "Marine invasive species: validation of citizen science and implications for national monitoring networks," Biological Invasions, vol. 10, no. 1, pp. 117-128, 2008.

[10] P. Gilbert, L. Cox, J. Jung, and D. Wetherall, "Toward trustworthy mobile sensing," in Proceedings of the Eleventh Workshop on Mobile Computing Systems \& Applications, 2010, pp. 31-36.

[11] I. Martí, L. Rodríguez, and M. Benedito, "Mobile application for noise pollution monitoring through gamification techniques," in ComputingICEC 2012, 2012, pp. 562-571.

[12] S. Sheppard and L. Terveen, "Quality is a verb: the operationalization of data quality in a citizen science community," in Proceedings of the 7th International Symposium on Wikis and Open Collaboration, 2011, pp. $29-38$.

[13] D. Brossard, B. Lewenstein, and R. Bonney, "Scientific knowledge and attitude change: The impact of a citizen science project," International Journal of Science Education, vol. 27, no. 9, pp. 1099-1121, 2005.

[14] D. Thau, R. Morris, and S. White, "Contemporary challenges in ambient data integration for biodiversity informatics," in On the Move to Meaningful Internet Systems: OTM 2009 Workshops, 2009, pp. 59-68.

[15] N. Van House, "Digital libraries and practices of trust: networked biodiversity information," Social Epistemology, vol. 16, no. 1, pp. 99$114,2002$.

[16] W. Hartwell and D. Shafer, "Public Involvement as an Element in Designing Environmental Monitoring Programs," Environmental Monitoring, pp. 169-184, 2011.

[17] K. Huang, S. Kanhere, and W. Hu, "Are you contributing trustworthy data?: the case for a reputation system in participatory sensing," in Proceedings of the 13th ACM international conference on Modeling, analysis, and simulation of wireless and mobile systems, 2010, pp. 14 22.

[18] R. Bonney, C. B. Cooper, J. Dickinson, S. Kelling, T. Phillips, K. V. Rosenberg, and J. Shirk, "Citizen science: a developing tool for expanding science knowledge and scientific literacy," BioScience, vol. 59, no. 11 , pp. $977-984,2009$.

[19] C. Borgman, "The conundrum of sharing research data," Journal of the American Society for Information ..., vol. 63, no. 6, pp. 1059-1078, 2012.

[20] J. Exner, P. Zeile, and B. Streich, "Urban Monitoring Laboratory: New Benefits and Potential for Urban Planning through the Use of Urban Sensing, Geo-and Mobile-Web," in Real CORP Proceedings, 2011, pp. 1087-1096.

[21] C. Lepczyk, "Integrating published data and citizen science to describe bird diversity across a landscape," Journal of Applied Ecology, vol. 42, no. 4, pp. 672-677, 2005.

[22] N. Davies, J. Landay, S. Hudson, and A. Schmidt, "Rapid prototyping for ubiquitous computing," IEEE Pervasive Computing, pp. 15-17, 2005.

[23] S. Moran, N. Pantidi, T. Rodden, A. Chamberlain, C. Griffiths, D. Zilli, G. Merrett, and A. Rogers, "Listening to the Forest and its Curators: Lessons Learnt from a Bioacoustic Smartphone Application Deployment," in Proceedings of the SIGCHI Conference on Human Factors in Computing Systems [In Press], 2014. 